\title{
Random-cluster correlation inequalities for Gibbs fields
}

\author{
Alberto Gandolfi \\ NYU Abu Dhabi \\ Dipartimento di Matematica e Informatica U. Dini \\ Università di Firenze
}

Wednesday $11^{\text {th }}$ April, 2018

\begin{abstract}
In this note we prove a correlation inequality for local variables of a Gibbs field based on the connectivity by active hyperbonds in a random cluster representation of the non overlap configuration distribution of two independent copies of the field.

As a consequence, we show that absence of Machta-Newman-Stein blue bonds percolation implies uniqueness of Gibbs distribution in EA Spin Glasses. In dimension two this could constitute a step towards a proof that the critical temperature is zero.
\end{abstract}

\section{Introduction}

The classic FK representation FK72 allows to express the spin spin correlation of Ising ferromagnets in terms of the FK occupied bonds connectivity in the random cluster representation of the Ising model. No similar expressions or bounds are available for correlation of more general observables, or even for spin correlations in general Gibbs models.

In this paper, we provide a general correlation inequality of this type by taking two copies of the Gibbs field. Specifically, the correlation of any two local observables is bounded by the active hyperbond (a generalization of the occupied bonds) connectivity in a (generalized) random cluster representation

\footnotetext{
1 AMS 2010 subject classifications. 60G60, 60J99, 82B44, 82D30.

Key words and phrases. Correlation inequalities, Gibbs fields, random cluster representation, disagreement percolation, Spin Glasses
} 
of the non overlap configuration distribution between two independent copies of a Gibbs field (called "foldings" in BG13]), the connectivity being averaged over the overlap configuration distribution. It is, to the knowledge of the author, the first result of this type with such a wide spectrum of applicability.

The overlap and non-overlap configuration distributions in two independent copies are obtained, for a general Gibbs measure, by fixing the value of the sum of the corresponding spins for each vertex: we declare an overlap if there is only one pair of spin values, one spin from each copy, whose sum is equal to the prescribed sum, and a non-overlap when there are more possibilities. For the special case in which the spins take only the values \pm 1 , as in Ising model or Spin Glasses, we have overlap when the two spins agree, and non-overlap when they disagree, in accordance with standard terminology about overlap in Spin Glass theory MNS08.

The use of the sum (and difference) of spins in two independent copies of an Ising model traces back to Percus and Lebowitz [L74, and the non-overlap configuration distribution is essential in [R00. A generalized random cluster representation (RCR) appears in BG13, and it is further generalized here. The use of product measure to prove inequalities appears also in G18.

Some consequences of the main inequality are then drawn in the paper; they include a criterium for extremality of a Gibbs distribution, which allows to retrieve the critical point for uniqueness of Markov Chains in a ferromagnetic Ising model on a binary tree; and a criterium for uniqueness of the Gibbs distribution, which is then compared with Dobrushin criterium and disagreement percolation (see Section 4).

Our results are then related, in Section 4.5, to the MNS representation of $\pm J$ spin glasses [MNS08; our final result is that absence of percolation of MNS blue bonds (in the non overlap region, and, hence, tout court absence of such percolation) for any boundary condition implies a.e. uniqueness of the Gibbs distribution. It is then conceivable that two dimensional geometric constraints prevent the formation of MNS blue bond percolation at any finite temperature, yielding a proof of the long standing conjecture that phase transition occurs at zero temperature in the two dimensional EA model (see [NS13], Page 84, or [TTC17, Page 48).

The author would like to thank J. van den Berg, C. Newman and D. Stein for very valuable discussions.

\section{Preliminaries}

\subsection{Overlap and non overlap configuration distributions}

We consider an infinite graph $\mathcal{G}=(V, E)$, and a locally finite family of hyperbonds $\mathcal{B}=\{b \subset V, b$ finite $\} ; \Lambda$ indicates finite subsets of $V$, and $\delta \Lambda$ is the set of vertices $v \in V$ such that there is a $b \in \mathcal{B}$ with $v \in b, b \cap \Lambda \neq \emptyset, b \cap \Lambda^{c} \neq \emptyset$.

We then take $\Omega=F^{V}$, with $F$ a finite alphabet; and for any $\Lambda \subset V$, $\Omega_{\Lambda}=F^{\Lambda}$. Combined configurations are denoted by juxtaposing symbols, so for 
disjont $C_{1}, C_{2}, \omega_{C_{1}} \omega_{C_{2}}$ is the configuration of $\Omega_{C_{1} \cup C_{2}}$ obtained from $\omega_{C_{1}}$ and $\omega_{C_{2}}$.

We then consider an interaction $\phi$ defined on $\cup_{b \in \mathcal{B}} \Omega_{b}$; to include possible constraints we allow $\phi=\infty$; although it would be more expressive to use a different collection of hyperbonds from $\mathcal{B}$ for possible constraints (see [GL16])), such a distinction is not needed for our purposes here.

The $\mathcal{B}$-Gibbs measure on $\Lambda$ with interactions $\phi$ and boundary conditions $\tilde{\omega}_{\Lambda^{c}}$ is

$$
\mu_{\phi, \Lambda, \tilde{\omega}_{\Lambda^{c}}}\left(\omega_{\Lambda}\right)=\frac{1}{Z} e^{\sum_{b \in \Lambda} \phi\left(\omega_{b}\right)+\sum_{b: b \cap \Lambda \neq \emptyset, b \cap \Lambda^{c} \neq \emptyset} \phi\left(\omega_{b \cap \Lambda} \tilde{\omega}_{b \cap \Lambda^{c}}\right)} .
$$

Thermodynamic limits in $\Lambda$ of $\mu_{\phi, \Lambda, \tilde{\omega}_{\Lambda^{c}}}$ are denoted by $\mu_{\phi}=\mu_{\phi, \tilde{\omega}}$, where $\tilde{\omega}$ denotes a sequence of boundary conditions.

We next consider two copies $\Lambda^{(1)}, \Lambda^{(2)}$ of $\Lambda$, and the product space $\Omega^{\prime}=$ $\Omega_{\Lambda^{(1)}} \times \Omega_{\Lambda^{(2)}}$ with the product measure $\mu_{\phi, \Lambda, \tilde{\omega}_{\Lambda^{c}}}^{(1)} \times \mu_{\phi, \Lambda, \tilde{\omega}_{\Lambda^{c}}}^{(2)}$, with $\mu_{\phi, \Lambda, \tilde{\omega}_{\Lambda^{c}}}^{(\ell)}={ }^{d}$ $\mu_{\phi, \Lambda, \tilde{\omega}_{\Lambda}}$, i.e. we consider two independent copies of the model.

We are interested in conditioning to the values of local functions with some invertibility. There are many possible choices, and for simplicity we restrict to the sum of spins, i.e. to $\sigma_{i}=\omega_{i}^{(1)}+\omega_{i}^{(2)}$, where $\omega^{(\ell)} \in \Omega^{(\ell)}$. Then, $\sigma=$ $\left\{\sigma_{i}\right\}_{i \in \Lambda} \in \Sigma=\prod_{i \in \Lambda} \tilde{F}$ with $\tilde{F}=\left\{a: a=a_{1}+a_{2}, a_{\ell} \in F\right\}$. Given $\sigma \in \Sigma$ and $\omega \in \Omega$, we denote by $\sigma-\omega$ the configuration such that $(\sigma-\omega)_{i}=\sigma_{i}-\omega_{i}$. Given $\sigma$, a probability $\mu$ on $\Omega$ is called $\sigma$-symmetric if $\mu(\omega)=\mu(\sigma-\omega)$ for all $\omega$ 's.

Given $\sigma$, let $W_{\sigma}=\left\{\left(\omega^{(1)}, \omega^{(2)}\right): \omega_{i}^{(1)}+\omega_{i}^{(2)}=\sigma_{i}\right.$ for all $\left.i \in \Lambda\right\}$. The collection $\left\{W_{\sigma}\right\}_{\sigma \in \Sigma}$ forms a partition of $\Omega^{\prime}$, and we define the overlap configuration distribution

$$
\rho_{\phi, \Lambda, \tilde{\omega}_{\Lambda^{c}}}(\sigma)=\left(\mu_{\phi, \Lambda, \tilde{\omega}_{\Lambda^{c}}}^{(1)} \times \mu_{\phi, \Lambda, \tilde{\omega}_{\Lambda} c}^{(2)}\right)\left(W_{\sigma}\right),
$$

and the non overlap configuration distribution

$$
\mu_{\phi, \Lambda, \tilde{\omega}_{\Lambda^{c}}}^{\sigma}(\omega)=\left(\mu_{\phi, \Lambda, \tilde{\omega}_{\Lambda^{c}}}^{(1)} \times \mu_{\phi, \Lambda, \tilde{\omega}_{\Lambda^{c}}}^{(2)}\right)((\omega, \sigma-\omega) \mid \sigma) .
$$

By definition, for an event $A \subseteq \Omega$,

$$
\begin{aligned}
\mu_{\phi, \Lambda, \tilde{\omega}_{\Lambda^{c}}}(A) & =\left(\mu_{\phi, \Lambda, \tilde{\omega}_{\Lambda^{c}}}^{(1)} \times \mu_{\phi, \Lambda, \tilde{\omega}_{\Lambda^{c}}}^{(2)}\right)(A \times \Omega) \\
& =\sum_{\sigma \in \Sigma} \mu_{\phi, \Lambda, \tilde{\omega}_{\Lambda^{c}}}^{\sigma}(A) \rho_{\phi . \Lambda, \tilde{\omega}_{\Lambda^{c}}}(\sigma) .
\end{aligned}
$$

In the special case that $\Omega=\{-1,1\}^{\Lambda}, \sigma_{i} \in\{-2,0,2\}$; moreover, conditioned on $\sigma_{i}, \sigma_{i}-\omega_{i}$ equals $-\omega_{i}$ if $\sigma_{i}=0$, and $+\omega_{i}$ otherwise. The overlap region is where $\sigma_{i} \neq 0$, as then only the pair $\left(\omega_{i}^{(1)}, \omega_{i}^{(2)}\right)$ is admissible with $\omega_{i}^{(j)}=\sigma_{i} / 2$ for both $j$ 's; and the nonoverlap region is where $\sigma_{i}=0$, in which case two pairs are admissible. This example justifies the reference to overlap in the names given to the two distributions above.

Given $\sigma$, let $\Omega(\sigma)=\prod_{i \in \Lambda} F\left(\sigma_{i}\right) \subseteq \Omega$, where $F\left(\sigma_{i}\right)=\{a \in F: \exists b \in$ $F$ with $\left.a+b=\sigma_{i}\right\}$. The region $K(\sigma)=\left\{i:\left|F\left(\sigma_{i}\right)\right|=1\right\}$ is the the overlap region, and the non overlap distribution is, in fact, a distribution on $K^{c}(\sigma)$ : in 
BG13 such distribution is called a "folding" of $\mu$, and foldings form a collection of distributions indexed by the overlap configuration $\sigma$. If the initial distribution is Gibbs, then the non overlap distribution is simmetrized Gibbs:

Lemma 2.1. Given a $\mathcal{B}(\Lambda)$-Gibbs distribution $\mu_{\phi, \Lambda, \tilde{\omega}_{\Lambda^{c}}}$ on $\Omega$ with interactions $\phi$, and given $\sigma \in \Sigma$, the non overlap configuration distribution $\mu_{\phi, \Lambda, \tilde{\omega}_{\Lambda c}}^{\sigma}$ is a $\sigma$ symmetric $\mathcal{B}(\Lambda)$-Gibbs distribution on $\Omega(\sigma)$ with interactions $\phi^{\prime}\left(\omega_{b}\right)=\phi\left(\omega_{b}\right)+$ $\phi\left(\sigma_{b}-\omega_{b}\right)$ and boundary conditions $\tilde{\omega}_{\Lambda^{c}}$.

Proof. Given $\sigma$, for $\omega \in \Omega(\sigma)$ we have

$$
\begin{aligned}
\mu_{\phi, \Lambda, \tilde{\omega}_{\Lambda^{c}}}^{\sigma}(\omega)= & \left(\mu_{\phi, \Lambda, \tilde{\omega}_{\Lambda^{c}}}^{(1)} \times \mu_{\phi, \Lambda, \tilde{\omega}_{\Lambda^{c}}}^{(2)}\right)((\omega, \sigma-\omega) \mid \sigma) \\
= & \frac{1}{Z^{\prime}} \exp \left(\sum_{b \in \Lambda}\left(\phi\left(\omega_{b}\right)+\phi\left(\sigma_{b}-\omega_{b}\right)\right)\right. \\
& \quad+\sum_{b: b \cap \Lambda \neq \emptyset, b \cap \Lambda^{c} \neq \emptyset} \phi\left(\omega_{b \cap \Lambda \Lambda} \tilde{\omega}_{b \cap \Lambda^{c}}\right)+\phi\left(\sigma_{b}-\omega_{b \cap \Lambda} \tilde{\omega}_{b \cap \Lambda^{c}}\right) \\
= & \frac{1}{Z^{\prime}} \exp \left(\sum_{b \in \Lambda} \phi^{\prime}\left(\omega_{b}\right)\right. \\
& \quad+\sum_{b: b \cap \Lambda \neq \emptyset, b \cap \Lambda^{c} \neq \emptyset} \phi^{\prime}\left(\omega_{b \cap \Lambda} \tilde{\omega}_{b \cap \Lambda^{c}}\right) \\
= & \mu_{\phi, \Lambda, \tilde{\omega}_{\Lambda^{c}}}^{\sigma}(\sigma-\omega) .
\end{aligned}
$$

Clearly, $\left(\mu_{\phi, \Lambda, \tilde{\omega}_{\Lambda^{c}}}^{(1)} \times \mu_{\phi, \Lambda, \tilde{\omega}_{\Lambda^{c}}}^{(2)}\right)((\omega, \sigma-\omega))=0$ for $\omega \notin \Omega(\sigma)$.

\subsection{Random cluster representations RCR's}

A $\mathcal{B}$-Random Cluster Representation or $\mathcal{B}$-RCR of a probability $\mu$ on $\Omega_{\Lambda}$ is a way of expressing $\mu$ using a base probability $\nu$ on the configurations $\eta \in H=\prod_{b \in \mathcal{B}} \mathcal{P}\left(\Omega_{b}\right)$, where $\mathcal{P}\left(\Omega_{b}\right)$ indicates the collection of all subsets of $\Omega_{b}$, as follows: for every $\omega \in \Omega_{\Lambda}$

$$
\mu(\omega)=\frac{1}{Z_{1}} \sum_{\eta \in H} \nu(\eta) \prod_{b \in \mathcal{B}} \mathbb{I}_{\omega_{b} \in \eta_{b}}=\frac{1}{Z_{1}} \sum_{\eta \in H, \eta \sim \omega} \nu(\eta)
$$

where $Z_{1}$ is a normalizing factor. Configurations in $H$, namely prescriptions of collections of local spin configurations, are called hyperbond variables, or simply bond variables when only pairs of spins are involved.

Note that the $\mathcal{B}$-RCR is not unique. Note also that (6) can be used to define new probabilities $\mu$ once $H$ and $\nu$ are given.

If $\mu=\mu_{\phi, \Lambda, \tilde{\omega}_{\Lambda^{c}}}$ is $\mathcal{B}$-Gibbs, with possible hard core constraints, then the following procedure produces a variety of $\mathcal{B}$-RCR's in which $\nu=\prod_{b \in \mathcal{B}} \nu_{b}$ is Bernoulli. For each $b$, consider the "energy" levels $\left\{e^{\phi\left(\omega_{b}\right)}\right\}_{\omega_{b} \in \Omega_{b}}=\left\{e^{\phi_{1}}, \ldots, e^{\phi_{k}}\right\}$, 
$\phi_{1}>\cdots>\phi_{k}$, and a collection of $k$ subsets $\eta_{b}^{(1)}, \ldots, \eta_{b}^{(k)}$ of $\Omega_{b}$ which, for simplicity, do not split energy levels, i.e. $\omega_{b} \in \eta_{b}^{(i)}$ and $\phi\left(\omega_{b}\right)=\phi\left(\omega_{b}^{\prime}\right)$ imply $\omega_{b}^{\prime} \in \eta_{b}^{(i)}$ (notice that for mathematical convenience there is a plus sign in the exponent). Then let $A=\left[a_{i, j}\right]$ be a $k \times k$ 0-1 matrix with $a_{i, j}$ the indicator that $\eta_{b}^{(j)}$ contains all the configurations with energy level $\phi_{i}$. If, for all $b$ 's, the problem

$$
A \vec{p}=c_{b}\left[e^{\phi_{i}}\right] \text { for some } c_{b} \in \mathbb{R}, \quad \sum_{j=1}^{k} p_{j}=1, p_{j} \geq 0,
$$

where $\vec{p}$ is the vector with components $p_{j}^{\prime} s$, can be solved, then we can take $\nu_{b}\left(\eta_{b}^{(j)}\right):=p_{j}$ and $\nu$ is the base of a $\mathcal{B}-\mathrm{RCR}$ of $\mu_{\phi, \Lambda, \tilde{\omega}_{\Lambda^{c}}}$.

One particular case of the above mechanism has been described in BG13, in which $\eta_{b}^{(i)}$ are taken to be monotone, i.e. $\omega_{b} \in \eta_{b}^{(i)}$ iff $\phi\left(\omega_{b}\right) \geq \phi_{i}$. In that case, $A$ is $0-1$ upper triangular; as the vector $\left[e^{\phi_{i}}\right]$ is monotone decreasing, there is a nonnegative solution to the above problem, which can be normalized to give $\nu_{b}\left(\eta_{b}^{(i)}\right)=\frac{e^{\phi_{i}}-e^{\phi_{i+1}}}{e^{\phi_{1}}}$, with $\phi_{k+1}=-\infty$.

\subsection{Typed RCR and MNS blue-red bonds}

To achieve some additional expressive power, a typed RCR can also be introduced, in which different types of hyperbond configurations are used. To keep things simple, we discuss the case of two types only. A two-typed $\mathcal{B}$-RCR of a probability $\mu$ is given by a pair of probabilities $\nu^{(a)}$ on $H^{(\alpha)}=H$ and $\nu^{(\beta)}$ on $H^{(b)}=H$ such that for every $\omega \in \Omega_{\Lambda}$

$$
\mu(\omega)=\frac{1}{Z_{2}} \sum_{\eta \in H} \nu^{(\alpha)}(\eta) \nu^{(\beta)}(\eta) \prod_{b \in \mathcal{B}} \mathbb{I}_{\omega_{b} \in \eta_{b}^{(\alpha)}} \mathbb{I}_{\omega_{b} \in \eta_{b}^{(\beta)}},
$$

where $Z_{2}$ is a normalizing factor; the above definition introduces some novelties when constraints are imposed on the possible values of $\eta^{(\alpha)}$ and $\eta^{(\beta)}$. The constrained linear problem (3) becomes then

$$
\begin{array}{r}
{\left[A^{(\alpha)} \vec{p}^{(\alpha)}\right]_{k}\left[A^{(\beta)} \vec{p}^{(\beta)}\right]_{k}=c_{b}\left[e^{-\phi_{k}}\right] \text { for some constant } c_{b},} \\
\sum_{j=1}^{k} p_{j}^{(\alpha)}=\sum_{j=1}^{k} p_{j}^{(\beta)}=1 ; \quad p_{j}^{(\alpha)}, p_{j}^{(\beta)} \geq 0,
\end{array}
$$

with possibly additional constraints on the entries of $p^{(1)}$ and $p^{(2)}$.

An example of typed RCR appears in [MNS08, with a different terminology, for the quenched distribution of two independent copies of EA Spin Glasses. In each copy, $V=\mathbb{Z}^{d}, \mathcal{B}(V)$ consists of n.n. pairs of vertices, $\{-1,1\}^{V}$ and

$$
\mu_{\mathbf{J}, \Lambda, \tilde{\omega}_{\Lambda^{c}}}\left(\omega_{\Lambda}\right)=\frac{1}{Z} e^{\sum_{\{i, j\}} J_{i, j} \omega_{i} \omega_{j}+\sum_{\{i, j\}, i \in \Lambda, j \notin \Lambda} J_{i, j} \omega_{i} \tilde{\omega}_{j}},
$$


where $J_{i, j}$ are i.i.d. symmetric r.v.'s taking values in $\pm J$, for some fixed $J>0$. The model is quenched, in the sense that a fixed value of $\mathbf{J}=\left\{J_{i, j}\right\}_{\{i, j\} \in \mathcal{B}(V)}$ is taken, and later averaged on the $\mathbf{J}$ 's. We consider then two copies of the space: $\Lambda=\Lambda^{(1)} \times \Lambda^{(2)}$, two identical copies; $\omega_{\Lambda}=\omega_{\Lambda^{(1)}} \times \omega_{\Lambda^{(2)}}$, the product of any pair of not necessarily identical configurations; $b=b^{(1)} \times b^{(2)}=\{i, j\} \times\{i, j\}$, two copies of the same bond; and

$$
\phi\left(\omega_{b}\right)=J_{i, j}\left(\omega_{i}^{(1)} \cdot \omega_{j}^{(1)}+\omega_{i}^{(2)} \cdot \omega_{j}^{(2)}\right),
$$

where we have indicated by a dot the actual products of the values of the two spin configurations.

For the product space above, one can produce a one typed RCR from energy levels as follows. The energy levels are $\phi_{1}=2 J_{i, j}, \phi_{2}=0, \phi_{3}=-2 J_{i, j}$; if $\Omega_{\phi_{i}}=\left\{\omega_{b}: \phi\left(\omega_{b}\right)=\phi_{i}\right\}$, then $\eta_{b}$ can take one of the values $\Omega_{\phi_{1}}, \Omega_{\phi_{1}} \cup \Omega_{\phi_{2}}$ or $\Omega_{b} ;$ and

$$
A=\left[\begin{array}{lll}
1 & 1 & 1 \\
0 & 1 & 1 \\
0 & 0 & 1
\end{array}\right], \quad \nu_{b}\left(\eta_{b}\right)=\left\{\begin{array}{l}
1-e^{-2 J_{i, j}} \quad \text { if } \eta_{b}=\Omega_{\phi_{1}} \\
e^{-2 J_{i, j}}-e^{-4 J_{i, j}} \quad \text { if } \eta_{b}=\Omega_{\phi_{1}} \cup \Omega_{\phi_{2}} \\
e^{-4 J_{i, j}} \quad \text { if } \eta_{b}=\Omega_{b} .
\end{array}\right.
$$

On the other hand, a two typed RCR can be obtained constraining $\nu^{(\alpha)}$ to single out only energy levels corresponding to two configurations $\omega_{\Lambda^{(1)}}$ and $\omega_{\Lambda^{(2)}}$ which agree with the coupling in both copies, i.e. such that $J_{i, j} \omega_{i}^{(1)} \omega_{j}^{(1)}=$ $J_{i, j} \omega_{i}^{(2)} \omega_{j}^{(2)}=1$; and $\nu^{(\beta)}$ to single out only the energy level corresponding to agreement with the coupling in exactly one of the two copies, i.e. such that i.e. $\omega_{i}^{(1)} \omega_{i}^{(2)} \omega_{j}^{(1)} \omega_{j}^{(2)}=-1$. In this case, $A^{(1)}=\left[\begin{array}{ll}1 & 1 \\ 0 & 1 \\ 0 & 1\end{array}\right]$ and $A^{(2)}=\left[\begin{array}{ll}0 & 1 \\ 1 & 1 \\ 0 & 1\end{array}\right]$; and (5) becomes

$$
\left[\begin{array}{c}
\left(p_{1}^{(\alpha)}(i, j)+p_{2}^{(\alpha)}(i, j)\right) p_{2}^{(\beta)}(i, j) \\
p_{2}^{(\alpha)}(i, j)\left(p_{1}^{(\beta)}(i, j)+p_{2}^{(\beta)}(i, j)\right) \\
p_{2}^{(\alpha)}(i, j) p_{2}^{(\beta)}(i, j)
\end{array}\right]=c\left[\begin{array}{c}
e^{2 J_{i, j}} \\
0 \\
e^{-2 J_{i, j}}
\end{array}\right]
$$

The only solution is $p_{1}^{(\alpha)}(i, j)=1-e^{-4 J_{i, j}}, p_{1}^{(\beta)}(i, j)=1-e^{-2 J_{i, j}}$, as indicated in MNS08; the two variables are called there blue and red bonds, respectively, each being present with probability $p_{1}^{(\alpha)}$ and $p_{1}^{(\beta)}$, respectively. This is a twotyped RCR of the $\mathcal{B}(\Lambda)$-Gibbs distribution of two independent quenched EA Spin Glass configurations as for $\mathbb{I}^{(\alpha)}=\mathbb{I}_{J_{i, j} \omega_{i}^{(1)} \omega_{j}^{(1)}=1} \mathbb{I}_{J_{i, j} \omega_{i}^{(2)} \omega_{j}^{(2)}=1}$ and $\mathbb{I}^{(\beta)}=$ 
$\mathbb{I}_{\omega_{i}^{(1)} \omega_{j}^{(1)} \omega_{i}^{(2)} \omega_{j}^{(2)}=-1}$ we have, with no boundary conditions,

$$
\begin{aligned}
& \sum_{\left(\eta^{(\alpha)}, \eta^{(\beta)}\right) \sim\left(\omega^{(1)}, \omega^{(2)}\right)} \nu^{(\alpha)}\left(\eta^{(\alpha)}\right) \nu^{(\beta)}\left(\eta^{(\beta)}\right) \\
& =\frac{1}{Z} \prod_{\{i, j\} \in \mathcal{B}(\Lambda)} p_{2}^{(\alpha)}(i, j)\left(1-\mathbb{I}^{(\alpha)}\right) p_{2}^{(\beta)}(i, j)\left(1-\mathbb{I}^{(\beta)}\right) \\
& =\frac{1}{Z} e^{-4 \sum_{\{i, j\}: \mathbb{I}(\alpha)}=1} J_{i, j}-2 \sum_{\{i, j\}: \mathbb{I}(\beta)=1} J_{i, j} \\
& =\frac{1}{Z} e^{\sum_{\{i, j\} \in \mathcal{B}(\Lambda)} J_{i, j} \omega_{i}^{(1)} \omega_{j}^{(1)}+J_{i, j} \omega_{i}^{(2)} \omega_{j}^{(2)}} \\
& =\left(\mu_{\mathbf{J}, \Lambda} \times \mu_{\mathbf{J}, \Lambda}\right)\left(\omega_{\Lambda}^{(1)}, \omega_{\Lambda}^{(1)}\right)
\end{aligned}
$$

where the equality before the last one is obtained by factoring out $\prod_{\{i, j\} \in \mathcal{B}(\Lambda)} 2 J_{i, j}$. Boundary conditions can easily be incorporated.

The bond variables $\eta^{(\alpha)}$ are called blue bonds, and the $\eta^{(\beta)}$ red bonds, in the MNS representation.

\subsection{Active hyperbond connectivity}

In a RCR, the joint distribution on spin and hyperbond variables is denoted by

$$
Q_{\phi, \Lambda, \tilde{\omega}_{\Lambda^{c}}}(\omega, \eta)=\frac{1}{Z_{1}} \nu(\eta) \prod_{b \in \mathcal{B}} \mathbb{I}_{\omega_{b} \in \eta_{b}}=\frac{1}{Z_{1}} \nu(\eta) \mathbb{I}_{\omega \sim \eta} .
$$

Then, the Random Cluster Probability $P=P_{\phi, \Lambda, \tilde{\omega}_{\Lambda} c}$ is the marginal on the hyperbond variables: $P_{\phi, \Lambda, \tilde{\omega}_{\Lambda^{c}}}(\eta)=\sum_{\omega \in \Omega} Q_{\phi, \Lambda, \tilde{\omega}_{\Lambda^{c}}}(\omega, \eta)$. Notice that random cluster probability is absolutely continuous w.r.t. $\nu$, with a Radon Nikodym derivative computable, in principle, in terms of the geometrical features which can described in terms of $\eta$ (this is where the factor $2^{\text {number of clusters }}$ appears in the original FK distribution).

The most relevant feature of the hyperbond configuration $\eta_{b}$ at $b$ is whether it puts some restrictions on the compatible configurations $\omega_{b}$ or not. Given $\eta \in H$, the hyperbonds $b$ for which $\eta_{b} \neq \Omega_{b}$ are called active. We say that two hyperbonds $b(1), b(2)$ are directly connected if $b(1) \cap b(2) \neq \emptyset$; and that two sets of vertices $\Lambda_{1}, \Lambda_{2}$ are connected by active hyperbonds if there is a chain of sequentially directly connected active hyperbonds, two of which have non empty intersections with $M_{1}, M_{2}$. We indicate this event by $\Lambda_{A} \stackrel{a c t}{\longleftrightarrow} \Lambda_{B}$. Connected active hyperbonds form clusters, which are at the origin of the name of "random cluster" representation.

In the original $F K$ representation of the ferromagnetic Ising model, it is in fact the connectivity by active (or "occupied" in the original formulation) bonds which is equivalent to the spin-spin correlations. More precisely, in the 
ferromagnetic Ising model with no external field,

$$
\left\langle\omega_{i} \omega_{j}\right\rangle-\left\langle\omega_{i}\right\rangle\left\langle\omega_{j}\right\rangle=P(i \stackrel{\text { act }}{\longleftrightarrow} j) .
$$

One could hope to have a similar results, or at least an upper bound of correlation in terms of connectivity, in greater generality. Unfortunately, for other Gibbs distributions (even for those which admit a directly extended version of the FK representation) or for events $A, B$ which depend on more than one spin, the analogous bound for covariances is not valid in general. Below, we make some explicit calculation on a very simple example: a nearest neightbor (n.n.) two body interaction model on three aligned binary spins; in the example, couplings favor minus spins on the left, and plus spins on the right, both couplings involving the middle spin; therefore, a natural RCR has no compatible active bonds, and active bonds connectivity is zero; on the other hand, covariances between extreme spins are still nonzero.

Example 1. Take $\Lambda=\{1,2,3\}, \Omega=\{-1,1\}^{\Lambda}$, a two body interaction Gibbs distribution $\mu$ with interaction $\phi$ defined by $\phi\left(\omega_{\{1,2\}}\right)=J_{12} \mathbb{I}_{\omega_{1}=\omega_{2}=-1}$ and $\phi\left(\omega_{\{2,3\}}\right)=$ $J_{23} \mathbb{I}_{\omega_{2}=\omega_{3}=1}$. We have

$$
\begin{aligned}
\Delta \mu=\mu\left(\omega_{1}=\omega_{3}=1\right)-\mu\left(\omega_{1}=1\right) \mu\left(\omega_{3}=1\right) & =\frac{\left(1-e^{J_{12}}\right)\left(1-e^{J_{23}}\right)}{\left(2\left(2+e^{J_{12}}+e^{J_{23}}\right)\right)^{2}} \\
& =\frac{\left(1-e^{J_{12}}\right)\left(1-e^{J_{23}}\right)}{Z^{2}}>0
\end{aligned}
$$

and $\operatorname{Cov}\left(\omega_{1}, \omega_{3}\right)=4 \Delta \mu$.

A RCR representation has base $\nu=\nu_{12} \times \nu_{23}$, with $\nu_{i j}$ concentrated on $\left\{\Omega_{\{i, j\}}, \Omega_{\{i, j\}}^{*}\right\}$ where $\Omega_{\{1,2\}}^{*}=\left\{\omega_{\{1,2\}}: \omega_{1}=\omega_{2}=-1\right\}$ and $\Omega_{\{2,3\}}^{*}=\left\{\omega_{\{2,3\}}\right.$ : $\left.\omega_{2}=\omega_{3}=1\right\}$; moreover, $\nu_{i j}\left(\Omega_{\{i, j\}}^{*}\right)=1-e^{-J_{i j}}$. In fact, for $\omega \in \Omega$

$$
\begin{aligned}
\sum_{\eta: \eta \sim \omega} \frac{\nu(\eta)}{\sum_{\omega^{\prime}, \eta^{\prime}} \nu\left(\eta^{\prime}\right) \mathbb{I}_{\eta^{\prime} \sim \omega^{\prime}}} & =\frac{e^{-J_{12} \mathbb{I}_{\left.\left(\omega_{1}=\omega_{2}=-1\right)^{c}-J_{23} \mathbb{I}_{\left(\omega_{2}=\omega_{3}=1\right.}\right)^{c}}}}{Z_{\nu}} \\
& =\frac{e^{J_{12} \mathbb{I}_{\left(\omega_{1}=\omega_{2}=-1\right)}+J_{23} \mathbb{I}_{\left(\omega_{2}=\omega_{3}=1\right)}}}{Z}=\mu(\omega) .
\end{aligned}
$$

On the other hand

$$
\begin{aligned}
P(1 \stackrel{\text { act }}{\longleftrightarrow} 3) & =\sum_{\eta: 1 \stackrel{\text { act }}{\longleftrightarrow} 3 \text { in } \eta} \nu(\eta) n_{\eta} \\
& =\nu\left(\Omega_{\{1,2\}}^{*}, \Omega_{\{2,3\}}^{*}\right) n_{\eta}=0
\end{aligned}
$$

as $n_{\eta}=|\{\omega \in \Omega: \omega \sim \eta\}|=0$ since $\Omega_{\{1,2\}}^{*} \cap \Omega_{\{2,3\}}^{*}=\emptyset$. So, $\left|\operatorname{Cov}\left(\omega_{1}, \omega_{3}\right)\right|>$ $|\Delta \mu|>0=P(1 \stackrel{\text { act }}{\longleftrightarrow} 3)$ and there is no upper bound of correlations in terms of connectivity. 
Clearly, there could be other RCR's of the same model for which a bound holds, but the example shows that this does happen in general; in particular, the example shows also that lack of correlation, and even more independence, does not follow from lack of active (hyper)bond connectivity. This is an issue in the theory of Spin Glasses, for instance, in which lack of FK bond connectivity does not imply uniqueness of Gibbs phase as it does in its ferromagnetic counterpart (see [N94]).

\subsection{RCR of non overlap configuration distribution and In- tegrated Random Cluster distribution of active hyper- bonds}

For these reasons, we resort to the non overlap configuration distributions $\mu_{\phi . \Lambda, \tilde{\omega}_{\Lambda^{c}}}^{\sigma}$, and to their own RCR's. As $\mu_{\phi . \Lambda, \tilde{\omega}_{\Lambda^{c}}}^{\sigma}$ is $\mathcal{B}(\Lambda)$-Gibbs distribution, the non overlap configuration distributions $\mu_{\phi . \Lambda, \tilde{\omega}_{\Lambda^{c}}}^{\sigma}$ is $\mathcal{B}(\Lambda)$-Gibbs on $\Omega(\sigma)$ by Lemma 2.1] therefore, the methods shown above produce RCR's for each $\mu_{\phi . \Lambda, \tilde{\omega}_{\Lambda^{c}}}^{\sigma}$. We obtain a collection of RCR's basis $\nu_{\phi . \Lambda, \tilde{\omega}_{\Lambda^{c}}}^{\sigma}$, and their related marginals $P_{\phi . \Lambda, \tilde{\omega}_{\Lambda^{c}}}^{\sigma}$ over hyperbond variables $\eta \in H^{\sigma} \stackrel{=}{=} \prod_{b \in \mathcal{B}} \Omega_{b}(\sigma)$, where $\Omega_{b}(\sigma)=\prod_{i \in b} F\left(\sigma_{i}\right)$.

Notice that, by Lemma 2.1 $\mu_{\phi . \Lambda, \tilde{\omega}_{\Lambda^{c}}}^{\sigma}$ is $\sigma$-symmetric. Then, the RCR's can also be taken $\sigma$-symmetric, in the sense that if $\omega_{b} \in \eta_{b}$ then also $\left(\sigma_{b}-\omega_{b}\right) \in \eta_{b}$; in fact, if $\nu$ is the base of a RCR of $\mu_{\phi . \Lambda, \tilde{\omega}_{\Lambda^{c}}}^{\sigma}$, also $\nu^{\prime}$ defined by $\nu^{\prime}\left(\eta_{b}\right)=$ $\left(\nu\left(\eta_{b}\right)+\nu\left(\sigma-\eta_{b}\right)\right) / 2$, where for a set of configurations $A \subseteq \Omega_{b}, \eta_{b}-A=\left\{\omega_{b}\right.$ : $\omega_{b}=\eta_{b}-\omega_{b}^{\prime}$ for some $\left.\omega_{b}^{\prime} \in \Omega_{b}\right\}$, is a RCR for $\mu_{\phi . \Lambda, \tilde{\omega}_{\Lambda} c}^{\sigma}$.

As the focus is on active and non active hyperbonds, we introduce now $H^{\prime}=\prod_{b \in \mathcal{B}(\Lambda)}\{0,1\}, 1$ standing for "active", and consider the map $\mathscr{A}: H \rightarrow H^{\prime}$

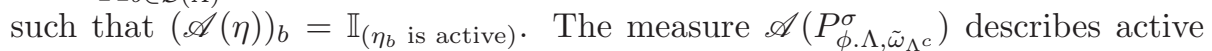
hyperbonds for the given $\sigma$, and we consider the Integrated Random Cluster distribution on active hyperbonds

$\bar{P}_{\phi . \Lambda, \tilde{\omega}_{\Lambda^{c}}}\left(\eta^{\prime}\right)=E_{\rho_{\phi . \Lambda, \tilde{\omega}_{\Lambda^{c}}}}\left(\mathscr{A}\left(P_{\phi . \Lambda, \tilde{\omega}_{\Lambda^{c}}}^{\sigma}\right)\right)\left(\eta^{\prime}\right)=\sum_{\sigma \in \Sigma} \mathscr{A}\left(P_{\phi \cdot \Lambda, \tilde{\omega}_{\Lambda^{c}}}^{\sigma}\right)\left(\eta^{\prime}\right) \rho_{\phi . \Lambda, \tilde{\omega}_{\Lambda^{c}}}(\sigma)$

defined on $H^{\prime}$.

The definition of $\bar{P}_{\phi . \Lambda, \tilde{\omega}_{\Lambda^{c}}}$ is such that if an hyperbond $b$ is fully included in the overlap region $K(\sigma)$ (in which there is only one pair of spin values satisfying the constraints), then $b$ is automatically non active; this means that (10) enhances the role of non active hyperbonds, thereby making the estimates of the next section more effective.

\section{Main results}

\subsection{Correlation inequality}

Our main result is a correlation inequality based on active hyperbond connectivity distributed according to the integrated random cluster probability $\bar{P}_{\phi . \Lambda, \tilde{\omega}_{\Lambda^{c}}}$. 
We have

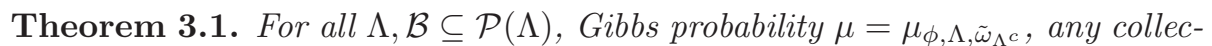
tion of Bernoulli $\mathcal{B}(\Lambda)-R C R$ 's $\left\{\nu^{\sigma}\right\}_{K \subseteq \Lambda, \alpha \in \Omega_{K}}$, and any two events $A, B \subseteq \mathcal{P}(\Omega)$ with supports $\Lambda_{A}, \Lambda_{B}$, respectively, we have

$$
|\mu(A \cap B)-\mu(A) \mu(B)| \leq \bar{P}_{\phi \cdot \Lambda, \tilde{\omega}_{\Lambda^{c}}}\left(\Lambda_{A} \stackrel{\text { act }}{\longleftrightarrow} \Lambda_{B}\right) .
$$

In words, the correlation between any pair of local events $A, B$ is bounded by the active hyperbond connectivity in RCR's of the non overlap configuration distributions, averaged over the overlap configuration.

Proof. We start from a preliminary argument about $\mu^{\sigma}$, the non overlap configuration distribution associated to $\mu=\mu_{\phi, \Lambda, \tilde{\omega}_{\Lambda} c}$. By Lemma 2.1. $\mu^{\sigma}$ is $\mathcal{B}(\Lambda)$-Gibbs for each $\sigma \in \Sigma$; by Section 2.2 and [BG13], it admits Bernoulli RCR's, so the assumptions make sense.

Given a configuration $\eta$ and a vertex $i \in V$, we let the cluster $C(i)$ be the set of vertices connected to $i$ by active hyperbonds (see Section 2.3), each possibly consisting of just one vertex. We denote such clusters by $C_{1}(\eta), \ldots, C_{t(\eta)}(\eta)$, with $\cup_{j=1}^{t(\eta)} C_{j}(\eta)=\Lambda$.

Suppose that $\eta$ is such that $\Lambda_{A} \stackrel{\text { aqt }}{\longleftrightarrow} \Lambda_{B}$, where $\Lambda_{A}$ and $\Lambda_{B}$ are the supports of the given $A$ and $B$; then for each $j, C_{j}(\eta)$ is connected to either $\Lambda_{A}$ or $\Lambda_{B}$, but not to both. Assume then that $C_{j}(\eta)$ is connected to $\Lambda_{A}$ for $j=1, \ldots, k$, and to $\Lambda_{B}$ for $j=k+1, \ldots, t(\eta)$, and let $C l(A)=C_{1}(\eta) \cup \ldots C_{k}(\eta)$ and $C l(B)=C_{k+1}(\eta) \cup \ldots C_{t}(\eta)(\eta)$ indicate the cluster of $A$, and of $B$, respectively. Then $\mathbb{I}_{\omega \in A \cap B}=\mathbb{I}_{\left(\omega_{C l(A)} \in A\right)} \mathbb{I}_{\left(\omega_{C l(B)} \in B\right)}$.

In addition, there are no active $b$ 's such that $b \cap C l(A) \neq \emptyset$ and $b \cap C l(B) \neq \emptyset$, so, for all such $b$ 's, $\mathbb{I}_{\left(\omega_{b} \in \eta_{b}\right)}=1$. This justifies the third equality in the next formula.

Next, recall that by the symmetry of the RCR, $\omega_{b} \in \eta_{b}$ if and only if $\sigma-\omega_{b} \in$ $\eta_{b}$. This justifies the fourth equality below.

We then have 


$$
\begin{aligned}
& \mu^{\sigma}(A \cap B)=\sum_{\omega \in A \cap B} \mu^{\sigma}(\omega) \\
& =\sum_{\omega \in A \cap B} \frac{1}{Z_{1}} \sum_{\eta \in H} \nu^{\sigma}(\eta) \mathbb{I}_{\eta \sim \omega} \\
& \leq \frac{1}{Z_{1}}\left(\sum_{\eta \in H^{\sigma}: \Lambda_{A} \stackrel{\text { act }}{\longleftrightarrow} \Lambda_{B}} \nu^{\sigma}(\eta) \sum_{\omega \in \Omega_{\Lambda}} \mathbb{I}_{\eta \sim \omega} \mathbb{I}_{\omega \in A \cap B}\right. \\
& \left.+\sum_{\eta \in H: \Lambda_{A} \stackrel{a c t}{\longleftrightarrow} \Lambda_{B}} \sum_{\omega \in \Omega_{\Lambda}} \nu^{\sigma}(\eta) \mathbb{I}_{\eta \sim \omega}\right)
\end{aligned}
$$

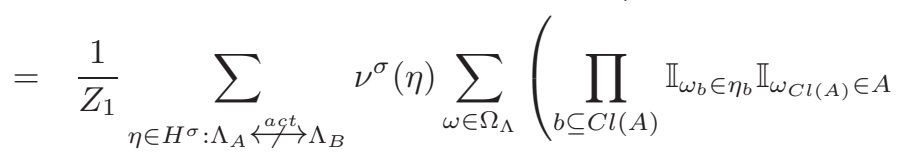

$$
\begin{aligned}
& \left.\prod_{b \subseteq C l(B)} \mathbb{I}_{\omega_{b} \in \eta_{b}} \mathbb{I}_{\omega_{C l(B)} \in B}\right) \\
& +\sum_{\eta \in H: \Lambda_{A} \stackrel{a c t}{\longleftrightarrow} \Lambda_{B}} P(\eta) \\
& =\frac{1}{Z_{1}} \sum_{\eta \in H^{\sigma}: \Lambda_{A} \stackrel{a c t}{\longleftrightarrow} \Lambda_{B}} \nu^{\sigma}(\eta) \sum_{\omega \in A \cap(\sigma-B)} \mathbb{I}_{\eta \sim \omega}+\sum_{\eta^{\prime} \in H^{\prime}: \Lambda_{A} \stackrel{a c t}{\longleftrightarrow} \Lambda_{B}} \mathscr{A}(P)\left(\eta^{\prime}\right) \\
& =\sum_{\omega \in A \cap(\sigma-B)} \frac{1}{Z_{1}} \sum_{\eta \in H} \nu^{\sigma}(\eta) \mathbb{I}_{\eta \sim \omega}+\mathscr{A}(P)\left(\Lambda_{A} \stackrel{\text { act }}{\longleftrightarrow} \Lambda_{B}\right) \\
& =\mu^{\sigma}(A \cap(\sigma-B))+\mathscr{A}(P)\left(\Lambda_{A} \stackrel{\text { act }}{\longleftrightarrow} \Lambda_{B}\right)
\end{aligned}
$$


Next, by denoting $\mu=\mu_{\phi, \Lambda, \tilde{\omega}_{\Lambda} c}$, we have

$$
\begin{aligned}
\mu(A \cap B) & =(\mu \times \mu)\left((A \cap B) \times \Omega_{\Lambda}\right) \\
& =\sum_{\sigma \in \Sigma}(\mu \times \mu)\left((A \cap B) \times \Omega \mid W_{\sigma}\right) \rho_{\phi . \Lambda, \tilde{\omega}_{\Lambda^{c}}}(\sigma) \\
& =\sum_{\sigma \in \Sigma} \mu_{\phi, \Lambda, \tilde{\omega}_{\Lambda^{c}}}^{\sigma}(A \cap B) \rho_{\phi . \Lambda, \tilde{\omega}_{\Lambda^{c}}}(\sigma) \\
& \leq \sum_{\sigma \in \Sigma}\left(\mu_{\phi, \Lambda, \tilde{\omega}_{\Lambda^{c}}}^{\sigma}(A \cap(\sigma-B))+\mathscr{A}(P)\left(\Lambda_{A} \stackrel{a c t}{\longleftrightarrow} \Lambda_{B}\right)\right) \rho_{\phi \cdot \Lambda, \tilde{\omega}_{\Lambda} c}(\sigma) \\
& =\sum_{\sigma \in \Sigma}(\mu \times \mu)\left((A \cap(\sigma-B)) \times \Omega \mid W_{\sigma}\right) \rho_{\phi . \Lambda, \tilde{\omega}_{\Lambda^{c}}}(\sigma)+\bar{P}_{\phi . \Lambda, \tilde{\omega}_{\Lambda^{c}}}\left(\Lambda_{A} \stackrel{a c t}{\longleftrightarrow} \Lambda_{B}\right) \\
& =\sum_{\sigma \in \Sigma}(\mu \times \mu)\left(A \times B \mid W_{\sigma}\right) \rho_{\phi . \Lambda, \tilde{\omega}_{\Lambda^{c}}}(\sigma)+\bar{P}_{\phi \cdot \Lambda, \tilde{\omega}_{\Lambda c}}\left(\Lambda_{A} \stackrel{a c t}{\longleftrightarrow} \Lambda_{B}\right) \\
& =\mu(A) \mu(B)+\bar{P}_{\phi \cdot \Lambda, \tilde{\omega}_{\Lambda} c}\left(\Lambda_{A} \stackrel{a c t}{\longleftrightarrow} \Lambda_{B}\right)
\end{aligned}
$$

The same relation holds when $B$ is replaced by $B^{c}$, and this proves (11).

Remark 1. Notice that a great number of choices has to be made in selecting a Bernoulli RCR of $\mu^{\sigma}$ for each $\sigma$, and the goodness of the bound depends on all of these choices. Clearly, one can get better bounds by selecting RCR's which use hyperbonds of small size (see Example Q below), or give high probability to non active hyperbond variables.

Remark 2. Notice also that the inequality in (12) depends on having removed the condition that $\omega \in A \cap B$ when $\Lambda_{A}$ is not actively connected to $\Lambda_{B}$ in $\eta$. Maintaining that condition would give an exact expression for the covariance of $A$ and $B$, but the connectivity event would no longer be measurable with respect to the $\eta$ variables (see Example 圆 below).

One can get a bound on the covariance of two local random variables by simply summing the previous on each pair of local configurations:

Corollary 3.2. With the assumptions of Theorem 3.1, and two random variables $X, Y$, replacing the events $A$ and $B$, depending on two disjoint finite sets $\Lambda_{X}$ and $\Lambda_{Y}$, respectively, one has

$$
|\operatorname{Cov}(X, Y)| \leq(|F|)^{\left|\Lambda_{X}\right|+\left|\Lambda_{Y}\right|} \bar{P}_{\phi . \Lambda, \tilde{\omega}_{\Lambda^{c}}}\left(\Lambda_{X} \stackrel{\text { act }}{\longleftrightarrow} \Lambda_{Y}\right)
$$

Example 2. Continuing Example 1. We apply Theorem 3.1 by conditioning on $\sigma \in \Sigma=\{-2,0,2\}^{\{1,2,3\}}$.

If $\sigma_{i} \neq 0$ for exactly one $i \in\{1,2,3\}$, then $|\Omega(\sigma)|=4$; $\mu^{\sigma}$, however, is symmetric under flip of the remaining spins, i.e. those located at $j$ and $k$, with $j \neq k, j \neq i \neq k$, and, therefore, only two parameters are needed, one for $\omega_{j}=\omega_{k}$ and the other for the case $\omega_{j}=-\omega_{k}$. 
If $\sigma=(0,2,0)$, however, $\mu^{\sigma}(\omega)=\frac{1}{Z} e^{J_{23}\left(\omega_{3}^{(1)}+\omega_{3}^{(2)}\right)}=\frac{1}{Z}$. So, only one parameter is needed; this can be realized with a RCR having just a field term (i.e. bonds of size 1$)$, and $P^{\sigma}(1 \stackrel{\text { act }}{\longleftrightarrow} 3)=0$. The same occurs for $\sigma=(0,-2,0)$.

If $\sigma=(2,0,0)$, then the $R C R$ of $\mu^{\sigma}$ has an active bond $\eta_{2,3}$, but that does not connect 1 and 3 , so again $P^{\sigma}(1 \stackrel{\text { act }}{\longleftrightarrow} 3)=0$. The same occurs if $\left\{i: \sigma_{i} \neq\right.$ $0\}=\{1\}$ or $\{3\}$.

If $\sigma_{i} \neq 0$ for more than one $i$, then no active bond is needed, as $\mu^{\sigma}$ is binary and symmetric.

This leaves then only one interesting case, namely the configuration $\tilde{\sigma}$ such that $\tilde{\sigma}_{i} \equiv 0$. In this case, for $\omega=\omega^{(1)}$, we have

$$
\begin{aligned}
\mu^{\tilde{\sigma}}(\omega) & =\mu \times \mu\left(\left(\omega^{(1)},-\omega^{(1)}\right) \mid W_{\tilde{\sigma}}\right) \\
& =\frac{1}{Z_{\tilde{\sigma}}} e^{J_{12}\left(\mathbb{I}_{\left(\omega_{1}^{(1)}=\omega_{2}^{(1)}=-1\right)}+\mathbb{I}_{\left(\omega_{1}^{(2)}=\omega_{2}^{(2)}=-1\right)}\right)+J_{23}\left(\mathbb{I}_{\left(\omega_{2}^{(1)}=\omega_{3}^{(1)}=1\right)}+\mathbb{I}_{\left(\omega_{2}^{(2)}=\omega_{3}^{(2)}=1\right)}\right)} \\
& =\frac{1}{Z_{\tilde{\sigma}}} e^{J_{12 \mathbb{I}} \mathbb{I}_{\left(\omega_{1}^{(1)}=\omega_{2}^{(1)}\right)}+J_{23} \mathbb{I}{ }_{\left(\omega_{2}^{(1)}=\omega_{3}^{(1)}\right)}}
\end{aligned}
$$

and $\rho\left(W_{\tilde{\sigma}}\right)=\frac{2\left(e^{J_{12}+J_{23}}+e^{J_{12}}+e^{J_{23}}+1\right)}{Z^{2}}=\frac{Z_{\tilde{\sigma}}}{Z^{2}}$. A Bernoulli RCR of $\mu^{\tilde{\sigma}}$ can now be obtained by taking base $\nu^{\tilde{\sigma}}=\nu_{12}^{\tilde{\sigma}} \times \nu_{23}^{\tilde{\sigma}}$, with $\nu_{i j}^{\tilde{\sigma}}$ concentrated on $\left\{\Omega_{\{i, j\}}, \Omega_{\{i, j\}}^{*}\right\}$ where $\Omega_{\{i, j\}}^{*}=\left\{\omega_{\{i, j\}}: \omega_{i}=\omega_{j}\right\}$, and moreover, $\nu_{i j}^{\tilde{\sigma}}\left(\Omega_{\{i, j\}}^{*}\right)=1-e^{-J_{i j}}$. In fact, for $\omega \in \Omega(\tilde{\sigma})$

$$
\begin{aligned}
\sum_{\eta: \eta \sim \omega} \frac{\nu^{\tilde{\sigma}}(\eta)}{\sum_{\omega^{\prime}, \eta^{\prime}} \nu^{\tilde{\sigma}}\left(\eta^{\prime}\right) \mathbb{I}_{\eta^{\prime} \sim \omega^{\prime}}} & =\frac{e^{-J_{12} \mathbb{I}_{\left(\omega_{1} \neq \omega_{2}\right)}-J_{23} \mathbb{I}_{\left(\omega_{2} \neq \omega_{3}\right)}}}{Z_{\nu^{\tilde{\sigma}}}} \\
& =\frac{e^{J_{12} \mathbb{I}_{\left(\omega_{1}=\omega_{2}\right)}+J_{23} \mathbb{I}_{\left(\omega_{2}=\omega_{3}\right)}}}{Z_{\tilde{\sigma}}}=\mu^{\tilde{\sigma}}(\omega),
\end{aligned}
$$

as $Z_{\nu_{\tilde{\sigma}}}=Z_{\tilde{\sigma}} / e^{J_{12}+J_{23}}$. Notice that this is almost the same representation as for the one single copy in Example [, but now the interaction has been symmetrized.

We now have

$$
\begin{aligned}
P^{\tilde{\sigma}}(1 \stackrel{a c t}{\longleftrightarrow} 3) & =\frac{1}{Z_{\tilde{\sigma}}} \sum_{\eta: 1 \stackrel{a c t}{\longleftrightarrow} 3 \text { in } \eta} \nu^{\tilde{\sigma}}(\eta)|\{\omega: \omega \sim \eta\}| \\
& =\frac{1}{Z_{\tilde{\sigma}}} 2 \nu_{12}^{\tilde{\sigma}}\left(\Omega_{1,2}^{*}\right) \nu_{23}^{\tilde{\sigma}}\left(\Omega_{2,3}^{*}\right) \\
& =\frac{1}{Z_{\tilde{\sigma}}} 2\left(1-e^{-J_{12}}\right)\left(1-e^{-J_{23}}\right) .
\end{aligned}
$$

Finally, using the value of $\Delta(\mu) \mu\left(\omega_{1}=\omega_{3}=1\right)-\mu\left(\omega_{1}=1\right) \mu\left(\omega_{3}=1\right)$ computed in Example 1,

$$
\begin{aligned}
\bar{P}(1 \stackrel{\text { act }}{\longleftrightarrow} 3) & =P^{\tilde{\sigma}}(1 \stackrel{a c t}{\longleftrightarrow} 3) \rho\left(W_{\tilde{\sigma}}\right) \\
& =e^{J_{12}+J_{23}} \frac{1}{Z_{\tilde{\sigma}}} 2\left(1-e^{-J_{12}}\right)\left(1-e^{-J_{23}}\right) \frac{Z_{\tilde{\sigma}}}{Z^{2}} \\
& =\frac{2\left(1-e^{-J_{12}}\right)\left(1-e^{-J_{23}}\right)}{Z^{2}}=2|\Delta(\mu)|,
\end{aligned}
$$


which is the inequality of Theorem 3.1.

Using next the spin spin covariance computed in Example 1, we have

$$
\left|\operatorname{Cov}\left(\omega_{1}, \omega_{3}\right)\right|=4|\Delta(\mu)| \leq 4 P^{\tilde{\sigma}}(1 \stackrel{\text { act }}{\longleftrightarrow} 3),
$$

which is the bound described in Corollary 3.2.

The bounds above are not sharp for the reasons mentioned in Remark 2 , and would become equalities if the conditions on $\omega_{1}=\omega_{3}=1$ were kept.

\subsection{Conditions for extremality and uniqueness of Gibbs phases}

Corollary 3.3. Suppose that for a sequence of b.c.'s $\left\{\tilde{\omega}_{\Lambda^{c}}\right\}_{\Lambda}$, such that the finite volume Gibbs measures $\mu_{\phi, \Lambda, \tilde{\omega}_{\Lambda^{c}}}$ converge weakly as $\Lambda$ diverges along a specific sequence, the following occurs: for each $\Lambda_{0} \subset V$ and $\epsilon>0$ there are $\Lambda_{1}, \Lambda_{2}$ such that

$$
\bar{P}_{\phi, \Lambda_{3}, \tilde{\omega}_{\Lambda_{3}^{c}}}\left(\Lambda_{0} \stackrel{a c t}{\longleftrightarrow} \delta \Lambda_{1}\right) \leq \epsilon
$$

or, alternatively,

$$
p_{\epsilon}=\rho_{\phi, \Lambda_{3}, \tilde{\omega}_{\Lambda_{3}^{c}}}\left(P_{\phi, \Lambda_{3}, \tilde{\omega}_{\Lambda_{3}^{c}}}^{\sigma}\left(\Lambda_{0} \stackrel{a c t}{\longleftrightarrow} \delta \Lambda_{1}\right) \leq \epsilon\right) \geq 1-\epsilon
$$

for all $\Lambda_{3} \supseteq \Lambda_{2}$ in the sequence of $\Lambda$ 's. Then the weak limit $\mu$ of $\mu_{\phi, \Lambda, \tilde{\omega}_{\Lambda^{c}}}$ is extremal.

Proof. (a) Consider the weak limit $\mu$ of $\mu_{\phi, \Lambda, \tilde{\omega}_{\Lambda^{c}}}$. Consider an event $A$ with finite support $\Lambda_{A}=\Lambda_{0}$ and take $\epsilon>0$, and $\Lambda_{1}$ and $\Lambda_{2}$ as in the hypothesis; then for any event $B$ with support $\Lambda_{B}=\Lambda_{4} \subseteq \Lambda_{2}$ such that $\Lambda_{4} \cap \Lambda_{1}=\emptyset$ we have

$$
\begin{aligned}
|\mu(A \cap B)-\mu(A) \mu(B)| & \leq \bar{P}_{\phi, \Lambda_{3}, \tilde{\omega}_{\Lambda_{3}^{c}}}\left(\Lambda_{A} \stackrel{a c t}{\longleftrightarrow} \Lambda_{B}\right) \\
& \leq \bar{P}_{\phi, \Lambda_{3}, \tilde{\omega}_{\Lambda_{3}^{c}}}\left(\Lambda_{0} \stackrel{a c t}{\longleftrightarrow} \delta \Lambda_{1}\right) \leq \epsilon
\end{aligned}
$$

for all $\Lambda_{3} \supseteq \Lambda_{2}$. Hence, the $\sigma$-algebra at infinity is trivial, which implies extremality of $\mu$ in the set $K_{\phi}$ of Gibbs states for $\phi$ (see, e.g., Theorem 1.11

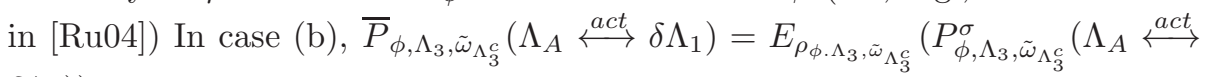
$\left.\left.\delta \Lambda_{1}\right)\right)$

$\leq\left(\epsilon p_{\epsilon}+\left(1-p_{\epsilon}\right)\right) \leq 2 \epsilon$, so that case (a) applies.

We also get a condition for uniqueness of the Gibbs state if the condition above holds for all possible sequences of b.c.'s since $K_{\phi}$ is convex and each element of $K_{\phi}$ would then be extremal. We thus have

Corollary 3.4. If the conditions of Corollary 3.3 hold for all sequences of b.c.'s $\left\{\tilde{\omega}_{\Lambda^{c}}\right\}_{\Lambda}$, then the Gibbs state is unique. 
To avoid technicalities the above results are stated in terms of finite volume distributions, but their corresponding infinite volume statements would be that absence of percolation of the RCR active hyperbonds in the non overlap configuration distribution with probability one with respect to the distribution of the overlap configuration implies uniqueness of the Gibbs state. When sufficient conditions for such absence of percolation are expressed locally, then our current condition closely resembles others present in the literature. Section 4 discusses these connections. One local condition is as follows:

Corollary 3.5. Consider the field $X_{b}^{\left(\phi, \Lambda, \tilde{\omega}_{\Lambda^{c}}\right)}, b \in \mathcal{B}$, given by $X_{b}^{\left(\phi, \Lambda, \tilde{\omega}_{\Lambda^{c}}\right)}(\eta)=$ $\mathbb{I}_{\left(\eta_{b} \text { is active) }\right.}$ when $\eta$ is distributed according to $\bar{P}_{\phi, \Lambda, \tilde{\omega}_{\Lambda^{c}}}$ on $\{b: b \cap \Lambda \neq \emptyset\}$. If, for a sequence of b.c.'s $\tilde{\omega}_{\Lambda^{c}}$ and each weak limit in $\Lambda \rightarrow V$, the field $X_{b}^{\left(\phi, \Lambda, \tilde{\omega}_{\Lambda^{c}}\right)}$ is stochastically dominated by hyperbond occupation variables distributed according to a Bernoulli probability $\tilde{P}$ on the Borel $\sigma$-algebra of $\prod_{b \in \mathcal{B}}\{0,1\}$, and there is no percolation of occupied hyperbonds for $\tilde{P}$, then each weak limit of $\mu_{\phi, \Lambda, \tilde{\omega}_{\Lambda} c}$ 's is extremal.

If this happens for all b.c.'s then the Gibbs distribution is unique.

A sufficient condition for the above domination is that

$$
p_{b}=\sup _{\eta_{\mathcal{B} \backslash b}} \bar{P}_{\phi, \Lambda, \tilde{\omega}_{\Lambda c}}\left(\eta_{b} \text { active } \mid \eta_{\mathcal{B} \backslash b}\right)
$$

is such that there is no percolation of occupied hyperbonds when they are independently selected with probability $p_{b}$.

Proof. If there is no percolation in $\tilde{P}$ then $\tilde{P}\left(\Lambda_{0} \stackrel{\text { occupied }}{\longleftrightarrow} \delta \Lambda_{1}\right) \rightarrow 0$ as $\Lambda_{1}$ diverges. By stochastic domination, $\tilde{P}\left(\Lambda_{0} \stackrel{\text { act }}{\longleftrightarrow} \delta \Lambda_{1}\right) \rightarrow 0$ so that (17) holds, and Corollaries 3.3 and 3.4 imply the first two statements.

The last statement follows from standard arguments in percolation theory (see, e.g. B93, Corollary 1), as (14) implies stochastic domination of $\bar{P}_{\phi, \Lambda, \tilde{\omega}_{\Lambda} c}$ by a probability $\tilde{P}$ in which occupied hyperbonds are independently selected with probability $p_{b}$.

\section{Applications and related works}

\subsection{Disagreement percolation and other uniqueness cri- teria}

A criterium for uniqueness of Gibbs distribution has been introduced by Dobrushin D68 (see also DS85, S79]), closely related to the sufficient condition of Corollary 3.5, in some cases our method performs better (see below).

Two copies have been considered in the works on disagreement percolation in BM94, BS94, B93. In the last paper there is also a correlation inequality based on two copies (see Th. 2.4 in BS94, but it involves site percolation and only holds for the hardcore model (see below). 
It is, however, interesting to relate our work to disagreement percolation in more details. In its first version B93, two independent configurations were selected, and one would focus on percolation of regions of disagreement or, equivalently, non overlap. The main result of [B93] is that absence of disagreement percolation for two Gibbs measures implies that they coincide. As proven in the next lemma, absence of disagreement percolation implies that there is a vanishing connectivity by active RCR hyperbonds in the non overlap region for any overlap configuration, as there is no connectivity by non overlap regions in the first place, so Corollary 3.4 implies uniqueness of the Gibbs phase: in this respect our results uniformly improve upon the first version of disagreement percolation.

Lemma 4.1. If for all pairs of Gibbs measures $\mu$ and $\mu^{\prime}$ in $K_{\phi}$, the probability $\mu \times \mu^{\prime}\left(\left(\omega, \omega^{\prime}\right)\right.$ : there is an infinite path of disagreement $)=0$, then condition $(b)$ of Corollary 3.3 holds for all sequences of b.c.'s $\left\{\tilde{\omega}_{\Lambda^{c}}\right\}_{\Lambda}$, and hence the Gibbs distribution is unique.

Proof. If condition (b) of Corollary 3.3 does not hold then there exist a sequence of b.c.'s $\left\{\tilde{\omega}_{\Lambda^{c}}\right\}_{\Lambda}$, a set $\Lambda_{0} \subset V$, and $\epsilon>0$ such that for all $\Lambda_{1}, \Lambda_{2}, \Lambda_{1} \subseteq \Lambda_{2}$,

$$
p_{\epsilon}=\rho_{\phi, \Lambda_{3}, \tilde{\omega}_{\Lambda_{3}^{c}}}\left(P_{\phi, \Lambda_{3}, \tilde{\omega}_{\Lambda_{3}^{c}}}\left(\Lambda_{0} \stackrel{a c t}{\longleftrightarrow} \delta \Lambda_{1}\right) \geq \epsilon\right) \geq \epsilon
$$

for some $\Lambda_{3} \supseteq \Lambda_{2}$.

Notice that if, for some $\sigma, \Lambda_{0} \stackrel{\text { act }}{\longleftrightarrow} \delta \Lambda_{1}$ in some $\eta$, then necessarily there is a path of disagreement between $\Lambda_{0}$ and $\delta \Lambda_{1}$ in all the configurations $\left(\omega_{\Lambda_{3}}, \omega_{\Lambda_{3}}^{\prime}\right) \in$ $W_{\sigma}$ which are compatible with $\eta$. Let

$$
D\left(\Lambda_{0}, \delta \Lambda_{1}\right)=\left\{\text { there is a path of disagreement between } \Lambda_{0} \text { and } \delta \Lambda_{1}\right\},
$$

then

$$
\begin{aligned}
& \left(\mu_{\phi, \Lambda_{3}, \tilde{\omega}_{\Lambda_{3}^{c}}} \times \mu_{\phi, \Lambda_{3}, \tilde{\omega}_{\Lambda_{3}^{c}}}\right)\left(D\left(\Lambda_{0}, \delta \Lambda_{1}\right)\right)=E_{\rho_{\phi, \Lambda_{3}, \tilde{\omega}_{\Lambda}^{c}}}\left(\mu_{\phi, \Lambda_{3}, \tilde{\omega}_{\Lambda_{3}^{c}}}^{\sigma}\left(D\left(\Lambda_{0}, \delta \Lambda_{1}\right)\right)\right. \\
& \geq E_{\rho_{\phi, \Lambda_{3}, \tilde{\omega}_{\Lambda_{3}}}}\left(P_{\Lambda_{3}, \phi}^{\sigma}\left(\Lambda_{0} \stackrel{a c t}{\longleftrightarrow} \delta \Lambda_{1}\right)\right) \\
& \geq \epsilon^{2} \text {. }
\end{aligned}
$$

Next, consider configurations $\omega_{\Lambda_{3} \backslash \Lambda_{1}}, \omega_{\Lambda_{3} \backslash \Lambda_{1}}^{\prime}$ and the two boundary conditions $\omega_{\Lambda_{3} \backslash \Lambda_{1}} \tilde{\omega}_{\Lambda_{3}^{c}}$ and $\omega_{\Lambda_{3} \backslash \Lambda_{1}}^{\prime} \tilde{\omega}_{\Lambda_{3}^{c}}$ for Gibbs distributions in $\Lambda_{1}$, and consider

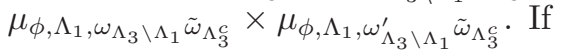

$$
\left(\mu_{\phi, \Lambda_{1}, \omega_{\Lambda_{3} \backslash \Lambda_{1}} \tilde{\omega}_{\Lambda_{3}^{c}}} \times \mu_{\phi, \Lambda_{1}, \omega_{\Lambda_{3} \backslash \Lambda_{1}} \tilde{\omega}_{\Lambda_{3}^{c}}}\right)\left(D\left(\Lambda_{0}, \delta \Lambda_{1}\right)\right)<\epsilon^{2}
$$

then by the Gibbs formula

$$
\mu_{\phi, \Lambda_{3}, \tilde{\omega}_{\Lambda_{3}^{c}}}=\int_{\Omega_{\Lambda_{3} \backslash \Lambda_{1}}} \mu_{\phi, \Lambda_{1}, \omega_{\Lambda_{3} \backslash \Lambda_{1}} \tilde{\omega}_{\Lambda^{c}}} \mu_{\phi, \Lambda_{3}, \tilde{\omega}_{\Lambda_{3}^{c}}}\left(d \omega_{\Lambda_{3} \backslash \Lambda_{1}}\right)
$$


we have that

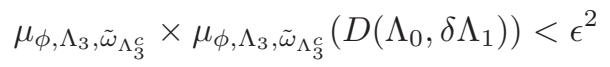

violating (18).

Therefore, there are configurations $\omega_{\Lambda_{3} \backslash \Lambda_{1}}, \omega_{\Lambda_{3} \backslash \Lambda_{1}}^{\prime}$ such that

$$
\left(\mu_{\phi, \Lambda_{1}, \omega_{\Lambda_{3} \backslash \Lambda_{1}} \tilde{\omega}_{\Lambda_{3}^{c}}} \times \mu_{\phi, \Lambda_{1}, \omega_{\Lambda_{3} \backslash \Lambda_{1}}^{\prime} \tilde{\omega}_{\Lambda_{3}^{c}}}\right)\left(D\left(\Lambda_{0}, \delta \Lambda_{1}\right)\right)>\epsilon
$$

for some $\Lambda_{3} \supseteq \Lambda_{2}$ for each $\Lambda_{2}$. By compactness and a diagonal argument, for

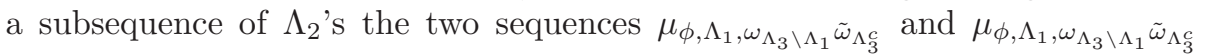
simultaneously converge in $K_{\phi}$ for all $\Lambda_{1}$, so their product converges to some product of Gibbs measures $\mu \times \mu^{\prime}$, for which $\left(\mu \times \mu^{\prime}\right)\left(D\left(\Lambda_{0}, \delta \Lambda_{1}\right)\right)>\epsilon^{2}>0$ for all $\Lambda_{1}$. Hence,

$$
\mu \times \mu^{\prime}\left(\left(\omega, \omega^{\prime}\right): \text { there is an infinite path of disagreement }\right)>\epsilon^{2},
$$

contradicting the assumptions.

It is interesting to notice that disagreement percolation is based on comparing distinct boundary conditions, while our method uses the same boundary conditions in the two copies.

The first version of disagreement percolation has been improved by using optimal couplings with respect to variational distance, instead of the independent coupling, BM94: there is no clear relation between the present RCR method and this improved version of disagreement percolation, but the RCR method presented here has a more explicit geometric interpretation, and in fact it also provides an explicit correlation bound.

\subsection{Hard core models and complete antiferromagnets}

Hard core models are discussed in BM94; they consist of a Gibbs measure on $\{0,1\}^{V}$ given by

$$
\mu_{a, \Lambda, \tilde{\omega}_{\Lambda^{c}}}\left(\omega_{\Lambda}\right)=\frac{1}{Z} a^{\omega_{i}} \prod_{\langle i, j\rangle} \mathbb{I}_{\omega_{i} \omega_{j}=0} \prod_{\langle i, j\rangle, i \in \Lambda, j \notin \Lambda} \mathbb{I}_{\omega_{i} \tilde{\omega}_{j}=0}
$$

i.e. 1's cannot be neighbor of each other. Let us assume that $V$ is bipartite into $V_{1}, V_{2}$. Then any for any overlap configuration $\sigma$ we must have that for each connected component $C$ of $\Lambda \backslash K, \omega_{i}=1$ for all $i \in V_{1} \cap C$, and $\omega_{i}=0$ for all $i \in$ $V_{2} \cap C$ or viceversa. So, $P^{\sigma}$ is concentrated on two configurations, and each bond of the graph is (at least part of) an active hyperbond. Hence, connectivity by active hyperbonds is equivalent in this case to connectivity by disagreement percolation in $\Lambda \backslash K$; our own criterium of Corollary 3.4 is equivalent to that of BS94 (see their Proposition 3.3 and Theorem 3.4), and also equivalent to the optimal coupling BM94 for this model. In particular, they all imply that there is uniqueness of the Gibbs phase if $a<\frac{p_{c}}{1-p_{c}}$, where $p_{c}$ is the critical point 
for site percolation on the graph. This estimate is better than the one obtained with the Dobrushin Shlossman method, so also our current one performs better than DS in this case.

For the complete antiferromagnet on $V=\mathbb{Z}^{d}$, disagreement percolation based on product coupling provided some improvement upon previous estimates B93. The use of optimal coupling has achieved a further improvement BM94, and so does our current integrated RCR method, which also requires percolation active bonds in the disagreement or non overlap regions. However, both optima coupling and integrated RCR do not change the zero temperature estimates, as the complete antiferromagnet tends to the hard core model as the temperature converges to zero, and there all estimates coincide, as discussed above.

\subsection{Ferromagnetic Ising model on the binary Cayley tree}

The ferromagnetic Ising model on the binary Cayley tree $(V, E)=T$ with couplings $J \geq 0$ and external field $h$, treated here as an illustration of detailed calculations, has configurations $\{-1,1\}^{V}$ and

$$
\mu_{(J, h), \Lambda, \tilde{\omega}_{\Lambda^{c}}}\left(\omega_{\Lambda}\right)=\frac{1}{Z} e^{\sum_{\langle i, j\rangle} J \omega_{i} \omega_{j}+\sum_{i \in \Lambda} h \omega_{i}+\sum_{\langle i, j\rangle, i \in \Lambda, j \notin \Lambda} J \omega_{i} \tilde{\omega}_{j}} .
$$

A detailed description of the phase diagram is in Ge88, Chapter 12. For $h(J)=\max _{t \geq 0}\left(\log \left(\frac{\cosh t+J}{\cosh t-J}\right)-t\right)$, if $J \leq \log 3 / 2$ and $h=h(J)=0$, or $J>$ $\log 3 / 2$ and $|h|>h(J)$, there is a unique Gibbs phase. We indicate by FK-RCR the Bernoulli RCR which corresponds to the original FK representation.

Some of the Gibbs distributions $\mu$ on the Cayley tree are Markov chains, in the sense that if $(i, j)$ is the oriented bond between two n.n. vertices $i, j \in T$, and $\mathcal{F}_{(-\infty, i)}$ is the $\sigma$-algebra generated by the vertices before $i$ in the order induced by $(i, j)$, then $\mu\left(\omega_{j} \mid \mathcal{F}_{(-\infty, i)}\right)=\mu\left(\omega_{j} \mid \omega_{i}\right)$ (see [Ge88]).

Corollary 4.2. There is no percolation of active bonds in the FK-RCR of active bonds in the non-overlap region of two independent copies of Markov chains on the binary Cayley tree if and only if all Markov Chains are extremal.

Proof. At given $J, h$, Markov chains on the binary Cayley tree are indexed by the solutions of $t=h+\log \left(\frac{\cosh t+J}{\cosh t-J}\right)$ and have transition matrix

$$
A=\left[a_{k, \ell}\right]=\left[\begin{array}{ll}
\frac{e^{J-t}}{2 \cosh (J-t)} & \frac{e^{t-J}}{2 \cosh (J-t)} \\
\frac{e^{-J-t}}{2 \cosh (J+t)} & \frac{e^{t+J}}{2 \cosh (J+t)}
\end{array}\right]
$$

for $k, \ell=0,1$ Ge88 Prop. 12.24.

Our extremality conditions of Corollary 3.3 imposed on the Markov chains give the exact calculation of the phase boundary line (although in general they are only sufficient conditions for uniqueness). In fact, a simple calculation shows that the marginal of the FK-RCR for the Ising model with $h=0$ field on a 
tree on the active bond variables is just a Bernoulli distribution, in which a bond is present with probability $p_{J}=\frac{\left(1-e^{-2 J}\right) / 2}{\left(1-e^{-2 J}\right) / 2+e^{-2 J}}=\tanh 2 J$; the same independence appears for the RCR in the non overlap region, but now $J$ is doubled, so a bond is active with probability $p_{J}^{N O}=\tanh 4 J$. If we condition on a region in the past being connected to the vertex $i$ in the $\mathrm{RCR}$ of the non overlap region, then $\omega_{i} \neq \omega_{i}^{\prime}$ in the two copies, and the forward process is independent of the past, given this information. In order for the bond $(i, j)$ to be active in the RCR of the non overlap region, it is necessary that also $\omega_{j} \neq \omega_{j}^{\prime}$, and that the bond is active, which occurs with probability $p_{J}^{N O}$.

Hence, for any $\Lambda_{0}$ in the past of $(i, j)$

$$
\bar{p}(J, h)=\bar{P}\left(\eta_{i, j} \text { is active } \mid \Lambda_{0} \stackrel{a c t}{\longleftrightarrow} i\right)=\left(a_{0,0} a_{11}-a_{1,0} a_{0,1}\right) \tanh 4 J,
$$

where $a_{i, j} \in A$. A condition for extremality of all the Markov chains is obtained, following Corollary 3.5 by a comparison with the critical point for independent percolation on the binary tree: $\bar{p}(J, h) \leq 1 / 2$. Some algebraic calculations show that this occurs exactly when $t=\arg \max _{t \geq 0}\left(\log \left(\frac{\cosh t+J}{\cosh t-J}\right)-t\right)$, hence at the value of $t$ which corresponds to the phase boundary line in $h$.

\subsection{Spin Glasses}

The Edwards Anderson Spin Glass model is defined as in (6). A RCR of (a single copy of) the EA Spin Glass model is discussed in [N94, and consists of $b=\{i, j\}$ for n.n. $i, j ; H=\left\{\{-1,1\}, \Omega_{b}\right\}$; and $\nu\left(\eta_{\{i, j\}}\right.$ active $)=p=1-e^{-2 J_{i, j}}$. Non frustration conditions appear in expressing the marginal $P$ on active bonds.

As, for each fixed overlap, the non overlap configuration distribution is also Gibbs of the same Spin Glass type, the representation above is also valid for the non overlaps, with doubled coupling. Additional representations for two quenched independent copies of EA Spin Glasses have been discussed in Section 2.3. in particular, we have seen that the MNS blue-red bond representation is a typed RCR. We see now that this representation can also be expressed in terms of overlap configurations, and that blue bonds are either in the overlap region, or in the non overlap region, and red bonds are in between, separating the two.

Partially numerical arguments in MNS08 suggest the formation of two large blue clusters (one in the overlap and one in the non overlap region) and that multiplicity of Gibbs state (with probability one with respect to the couplings) should appear when the two blue clusters have different densities.

Some interpretation of this behavior may come from the following consequence of our main result. Consider the joint distribution of the two typed (blue and red in the MNS model) RCR of the quenched EA Spin Glass

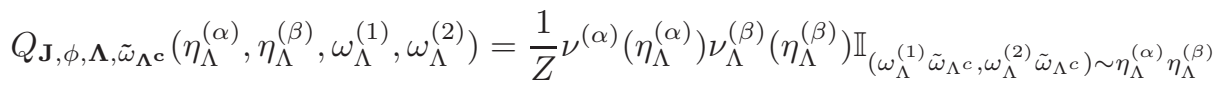

and for a sequence $\tilde{\omega}_{\Lambda^{c}}$ the (sub)sequential limits $Q_{\mathbf{J}, \phi}$ as $\Lambda \rightarrow \infty$. Let $A=$ (there is percolation of $\eta^{(\alpha)}$ active bonds $\{i, j\}$, s.t. $\omega_{i}^{(1)}=-\omega_{i}^{(2)}, \omega_{j}^{(1)}=-\omega_{j}^{(2)}$ ); 
$A$ corresponds to the event that there is percolation of MNS-blue bonds in the non overlap region of a pair of Spin Glass configurations.

Theorem 4.3. If, with probability one with respect to the coupling $\mathbf{J}$, for all sequences $\left\{\tilde{\omega}_{\Lambda^{c}}\right\}_{\Lambda}$, there is no MNS-blue bonds in the non overlap region, i.e. $Q_{\mathbf{J}, \phi}(A)=0$, then the Spin Glass Gibbs state is unique for a set of $\mathbf{J}$ 's of probability one.

Proof. Fix any finite set of vertices $\Lambda_{0}$. If $Q_{\mathbf{J}, \phi}(A)>0$ then it is a standard procedure in percolation theory to select appropriate configurations around $\Lambda_{0}$ such that percolation of $\eta^{(1)}$ active bonds in the non overlap region occurs from $\Lambda_{0}$ with positive probability. We thus assume that the $Q_{\mathbf{J}, \phi}$ probability of such percolation from $\Lambda_{0}$ is zero for almost all $\mathbf{J}$ 's. Then, for each such $\mathbf{J}$, and for each $\epsilon>0$, there are $\Lambda_{1}, \Lambda_{2}$ such that

$$
Q_{\mathbf{J}, \phi, \boldsymbol{\Lambda}_{\mathbf{3}}, \tilde{\omega}_{\Lambda_{3}}}\left(\Lambda_{0} \stackrel{a c t}{\longleftrightarrow} \delta \Lambda_{1}\right)<\epsilon
$$

for all $\Lambda_{3} \supseteq \Lambda_{2}$ in the sequence defining $Q_{\mathbf{J}, \phi}$.

Now, consider the map $\psi$ which transforms the variables representing MNSblue bonds within $\Lambda_{1}$ in the non overlap region of two configurations, namely $\left(\eta^{(\alpha)}, \omega^{(1)}, \omega^{(2)}\right)$ as defined in Section 2.3. into active bonds in the Bernoulli RCR of the non overlap configuration distribution, defined as follows. Let $\{i, j\}=$ $b \subseteq \Lambda_{1}$; with $\eta_{i, j}^{(\alpha)}$ having values either $\Omega_{\{i, j\}}^{(1)} \times \Omega_{\{i, j\}}^{(2)}$ or the set $\left\{\left(\omega^{(1)}, \omega^{(1)}\right)\right.$ : $\left.J_{i, j} \omega_{i}^{(1)} \omega_{j}^{(1)}=1, J_{i, j} \omega_{i}^{(2)} \omega_{j}^{(2)}=1\right\}$, then $\left(\psi\left(\eta_{\Lambda_{1}}^{(\alpha)}, \omega_{\Lambda_{1}}^{(1)}, \omega_{\Lambda_{1}}^{(2)}\right)\right)_{i, j}= \begin{cases}\Omega_{i, j} & \text { if } \eta_{i, j}^{(\alpha)}=\Omega_{\{i, j\}}^{(1)} \times \Omega_{\{i, j\}}^{(2)} \\ & \text { and } \omega_{i}^{(1)}=-\omega_{i}^{(2)}, \omega_{j}^{(1)}=-\omega_{j}^{(2)} . \\ \left\{\omega_{i, j}: J_{i, j} \omega_{i} \cdot \omega_{j}=1\right\} & \text { otherwise }\end{cases}$

We show here below that

$$
Q_{\mathbf{J}, \phi, \boldsymbol{\Lambda}_{\mathbf{3}}, \tilde{\omega}_{\boldsymbol{\Lambda}_{\mathbf{3}}}}\left(\eta_{\Lambda_{1}}^{(\alpha)}, \omega_{\Lambda_{1}}^{(1)}, \omega_{\Lambda_{1}}^{(2)}\right)=\bar{P}_{\mathbf{J}, \phi, \boldsymbol{\Lambda}_{\mathbf{3}}, \tilde{\omega}_{\mathbf{\Lambda}_{\mathbf{3}}^{\mathbf{c}}}}\left(\psi\left(\eta_{\Lambda_{1}}^{(\alpha)}, \omega_{\Lambda_{1}}^{(1)}, \omega_{\Lambda_{1}}^{(2)}\right)\right) .
$$

Then,

$$
\begin{aligned}
& \epsilon \geq Q_{\mathbf{J}, \phi, \boldsymbol{\Lambda}_{\mathbf{3}}, \tilde{\omega}_{\Lambda_{3}^{\mathrm{c}}}}\left(\Lambda_{0} \stackrel{a c t}{\longleftrightarrow} \delta \Lambda_{1}\right) \\
& =\bar{P}_{\mathbf{J}, \phi, \boldsymbol{\Lambda}_{3}, \tilde{\omega}_{\boldsymbol{\Lambda}_{3}^{\mathrm{c}}}}\left(\psi\left(\Lambda_{0} \stackrel{a c t}{\longleftrightarrow} \delta \Lambda_{1}\right)\right) \\
& =\bar{P}_{\mathbf{J}, \phi, \boldsymbol{\Lambda}_{\mathbf{3}}, \tilde{\omega}_{\Lambda_{3}^{\mathrm{c}}}}\left(\Lambda_{0} \stackrel{\text { act }}{\longleftrightarrow} \delta \Lambda_{1}\right)
\end{aligned}
$$

for all $\Lambda_{3} \supseteq \Lambda_{2}$, and hence by Part (a) of Corollary (3.3), the Gibbs state is unique.

To conclude, we have the following. For given $\sigma$ let $\Lambda_{\sigma}=\left\{i: \sigma_{1}=0\right\}$ and $\backslash \Lambda_{\sigma}=\mathcal{B}(\Lambda) \backslash \mathcal{B}\left(\Lambda_{\sigma}\right)$ 


$$
\begin{aligned}
& Q_{\mathbf{J}, \phi, \mathbf{\Lambda}_{\mathbf{3}}, \tilde{\omega}_{\Lambda_{\mathbf{3}}}}\left(\bar{\eta}_{\Lambda_{1}}^{(\alpha)}, \omega_{\Lambda_{1}}^{(1)}, \omega_{\Lambda_{1}}^{(2)}\right) \\
& \sum_{\eta_{\Lambda_{3}}^{(\alpha)}, \eta_{\Lambda_{3}}^{(\beta)}: \eta_{\Lambda_{1}}^{(\alpha)}=\bar{\eta}_{\Lambda_{1}}^{(\alpha)}} \\
& \sum_{\omega_{\Lambda_{3}}^{(1)}, \omega_{\Lambda_{3}}^{(2)}: \omega_{\Lambda_{1}}^{(1)}=-\omega_{\Lambda_{1}}^{(2)}} Q_{\mathbf{J}, \phi, \boldsymbol{\Lambda}_{3}, \tilde{\omega}_{\Lambda_{3}^{c}}}\left(\eta_{\Lambda_{3}}^{(\alpha)}, \eta_{\Lambda_{3}}^{(\beta)}, \omega_{\Lambda_{3}}^{(1)}, \omega_{\Lambda_{3}}^{(2)}\right) \\
& =\sum_{\eta_{\Lambda_{3}}^{(\alpha)}, \eta_{\Lambda_{3}}^{(\beta)}: \eta_{\Lambda_{1}}^{(\alpha)}=\bar{\eta}_{\Lambda_{1}}^{(\alpha)}} \\
& \sum_{\left(\omega_{\Lambda_{3}}^{(1)}, \omega_{\Lambda_{3}}^{(2)}\right) \sim\left(\eta_{\Lambda_{3}}^{(\alpha)}, \eta_{\Lambda_{3}}^{(\beta)}\right): \omega_{\Lambda_{1}}^{(1)}=-\omega_{\Lambda_{1}}^{(2)}} \frac{1}{Z} \nu^{(\alpha)}\left(\eta_{\Lambda_{3}}^{(\alpha)}\right) \nu_{\Lambda}^{(\beta)}\left(\eta_{\Lambda_{3}}^{(\beta)}\right) \\
& =\sum_{\sigma: \sigma_{\Lambda_{1}} \equiv 0} \frac{1}{Z} \prod_{\{i, j\} \subseteq \Lambda_{\sigma}}\left(1-e^{-4 J_{i, j}}\right) \mathbb{I}_{\bar{\eta}_{i, j} \text { (is active) }}+e^{-4 J_{i, j}} \mathbb{I}_{\bar{\eta}_{i, j} \text { (is not active) }} \\
& \sum_{\eta_{\Lambda_{\sigma}}^{(\alpha)}, \eta_{\backslash \Lambda_{\sigma}}^{(\beta)}\left\{(i, j\} \notin \Lambda_{\sigma}, \omega_{i}^{(1)} \cdot \omega_{i}^{(2)}=\omega_{j}^{(1)} \cdot \omega_{j}^{(2)}\right.}\left(1-e^{\left.-4 J_{i, j}\right)}\right) \mathbb{I}_{\bar{\eta}_{i, j}} \text { (is active) }+e^{-4 J_{i, j} j} \mathbb{I}_{\bar{\eta}_{i, j}} \text { (is not active) } \\
& \prod_{\left\{(i, j\} \unrhd \Lambda_{\sigma}, \omega_{i}^{(1)} \cdot \omega_{i}^{(2)}=-\omega_{j}^{(1)} \cdot \omega_{j}^{(2)}\right.}\left(1-e^{-2 J_{i, j}}\right) \mathbb{I}_{\bar{\eta}_{i, j}(\text { is active })}+e^{-2 J_{i, j}} \mathbb{I}_{\bar{\eta}_{i, j} \text { (is not active) }} \\
& \times\left|\left\{\left(\omega_{\Lambda_{3}}^{(1)}, \omega_{\Lambda_{3}}^{(2)}\right):\left(\omega_{\Lambda_{3}}^{(1)}, \omega_{\Lambda_{3}}^{(2)}\right) \sim \eta_{\Lambda_{\sigma}}^{(1)}, \eta_{\backslash \Lambda_{\sigma}}^{(1)}, \eta_{\backslash \Lambda_{\sigma}}^{(2)}\right\}\right| \\
& =\sum_{\sigma: \sigma_{\Lambda_{1}} \equiv 0} \frac{1}{Z} \prod_{\{i, j\} \subseteq \Lambda_{\sigma}}\left(1-e^{-4 J_{i, j}}\right) \mathbb{I}_{\bar{\eta}_{i, j} \text { (is active) }}+e^{-4 J_{i, j}} \mathbb{I}_{\bar{\eta}_{i, j} \text { (is not active) }} \\
& \times\left|\left\{\left(\omega_{\Lambda_{3}}^{(1)}, \omega_{\Lambda_{3}}^{(2)}\right):\left(\omega_{\Lambda_{3}}^{(1)}, \omega_{\Lambda_{3}}^{(2)}\right) \sim \eta_{\Lambda_{\sigma}}^{(1)}, \eta_{\backslash \Lambda_{\sigma}}^{(1)}, \eta_{\backslash \Lambda_{\sigma}}^{(2)}\right\}\right| \\
& \times \rho\left(\left(\omega_{\backslash \Lambda_{\sigma}}^{(1)}, \omega_{\backslash \Lambda_{\sigma}}^{(2)}\right): \omega_{i}^{(1)}=\omega_{i}^{(2)} \text { for all } i \in \Lambda_{3} \backslash \Lambda_{\sigma}\right) \\
& =E_{\rho}\left(\nu^{\rho}\left(\psi\left(\bar{\eta}_{\Lambda_{1}}^{(1)}, \omega_{\Lambda_{1}}^{(1)}, \omega_{\Lambda_{1}}^{(2)}\right)\right)\right. \\
& =\bar{P}\left(\psi\left(\bar{\eta}_{\Lambda_{1}}^{(1)}, \omega_{\Lambda_{1}}^{(1)}, \omega_{\Lambda_{1}}^{(2)}\right)\right. \text {; }
\end{aligned}
$$

the third equality follows from the fact that the sum is independent of $\left(\omega_{\Lambda_{3}}^{(1)}, \omega_{\Lambda_{3}}^{(2)}\right)$ as the cross interactions between the non overlap configuration in $\Lambda_{\sigma}$ and the overlap configuration in $\Lambda_{3} \backslash \Lambda_{\sigma}$ is always zero, since for $i \in \Lambda_{\sigma}$ and $j \notin \Lambda_{\sigma}$ we have $J_{i, j}\left(\omega_{i}^{(1)} \cdot \omega_{j}^{(1)}+\omega_{i}^{(2)} \cdot \omega_{j}^{(2)}\right)=J_{i, j}\left(\omega_{i}^{(1)} \cdot \omega_{j}^{(1)}-\omega_{i}^{(1)} \cdot \omega_{j}^{(1)}\right)=0$.

This finishes the proof.

The last theorem suggests that the observed unbalance in blue cluster densities at the phase transition could be caused by the onset of percolation of the blue cluster in the non overlap region. Restricting to dimension 2, it is conceivable that planar geometric constraints prevent the formation of a percolating 
blue cluster in the non overlap region and this could lead to a proof of the absence of phase transition at any finite temperature in the two-dimensional EA Spin Glasses.

\section{References}

[B93] van den Berg, J.: A Uniqueness Condition for Gibbs Measures, with Application to the 2-Dimensional Ising Antiferromagnet, Commun. Math. Phys. 152, 161-166 (1993).

[BG13] J. van den Berg, A. Gandolfi: BK-type inequalities and generalized randomcluster representations. PROBABILITY THEORY AND RELATED FIELDS (2013), Vol. 157, Issue 1-2, pp 157-181

[BM94] Van Den Berg, J., Maes, C.: "Disagreement Percolation in the Study of Markov Fields" Ann. Probab. Volume 22, Number 2 (1994), 749-763.

[BS94] Berg, J., van den, Steif, J.E.: "Percolation and the hard-core lattice gas model". Stochastic Processes and their Applications Volume 49, Issue 2, 179-197 (1994)

[D68] Dobrushin, R.L.: The problem of uniqueness of a Gibbs random field and the problem of phase transition. Funct. Anal. Appl. 2, 302-312 (1968)

[DS85] Dobrushin, R.L., Shlosman, S.B.: Constructive criterion for the uniqueness of a Gibbs field. In: Fritz, J., Jaffe, A., Szasz, D. (eds.), Statistical mechanics and dynamical systems. Boston: Birkhauser 1985, pp. 371-403.

[FK72] C.M. Fortuin and P.W. Kasteleyn, On the random-cluster model. I. Introduction and relation to other models, Physica 57, 536-564 (1972).

[G18] Gandolfi, A.: "FKG (and other inequalities) via (generalized) FK representation (and iterated folding)". Preprint (2018)

[GL16] Gandolfi, A., Lenarda, P.: "A note on Gibbs and Markov random fields with constraints and their moments", Math. and Mech. of Complex Systems, Vol. 4, No. 3-4, 407-422 (2016).

[Ge88] H.O. Georgii, Gibbs Measures and Phase Transitions, de Gruyter, Berlin (1988)

[G06] Grimmett, G.R.: The Random-Cluster Model. Springer, Berlin (2006)

[N94] C. Newman, "Disordered Ising systems and random cluster representations", Probability and Phase Transition (G. Grimmett, Ed.), Kluwer, Dordrecht, 1994, 247-260.

[MNS08] Machta, J., Newman, C.M., Stein, D.L.: The Percolation Signature of the Spin Glass Transition, J Stat Phys (2008) 130-113.

[L74] L. Lebowitz, J. L., "GHS and other inequalities", Comm. Math. Phys. Volume 35, Number 2 (1974), 87-92.

[NS13] Stein, D. L, Newman, C. M.: Spin Glasses and Complexity Princeton University Press (2013).

[R00] D. Reimer, Proof of the Van den Berg-Kesten Conjecture, Combinatorics, Probability and Computing 9, 27-32 (2000).

[Ru04] Ruelle, D.: Thermodynamic Formalism. Cambridge University Press, Cambridge (2004) 
[S79] Simon, B: "A remark on Dobrushin's uniqueness theorem", Comm. Math. Phys. Volume 68, Number 2 (1979), 183-185.

[TTC17] Tanaka, S., Tamura, R., Chakrabarti, B. K.: Quantum Spin Glasses, Annealing and Computation. Cambridge, UK: Cambridge University Press (2017).

Contact address: NYU Abu Dhabi Saadiyat Island P.O Box 129188 Abu Dhabi, $\mathrm{UAE}$

email: ag189@nyu.edu 